Rev. Per. Biol. 1980,2 (1): 3 - 7

\title{
ALGAS DEL SUELO DE LIMA Y ALREDEDORES
}

\author{
Reina Zúñiga A. \\ Programa Académico de Biología, Universidad Particular \\ "Ricardo Palma". Lima, Perú
}

\begin{abstract}
SUMARIO
Este trabajo trata sobre el estudio sistemático de algunas èspecies de Microcoleus, Noduuria, Cylindrospermum, Botrydium, Vaucheria y Stichococcus identificadas en muestras de suelo procedentes de algunos lugares de Lima y alrededores.
\end{abstract}

\section{SUMMARY}

This paper deals with the sistematic study of some species of Microcoleus, Nodularia, Cylindrospermum, Botrydium, Vaucheria and Stichococcus identified on soil samples from some places of Lima city and adjacent areas.

\section{INTRODUCCION}

Al revisar la bibliografía sobre las algas continentales de nuestro país, se comprueba que la mayoría de los estudios se refieren a las registradas en los diversos cuerpos de agua, ninguno trata en forma particular y exclusiva a las presentes en los ambientes terrestres.

El propósito de este trabajo, es el de dar a conocer sistemáticamente los diversos géneros y especies de algas que viven habitualmente sobre el suelo; con tal fin, se ha estudiado algunas muestras conservadas en el Herbario San Marcos (USM) del Museo de Historia Natural "Javier Prado", las mismas que fueron colectadas en diferentes lugares de la Ciudad de Lima $\mathrm{y}$ alrededores.

Se ha identificado las siguientes especies: Microcoleus paludosus (Kutzing) Gomont; Nodularia spumigena Mertens ex Born. et Flah; Cylindrospermum licheniforme Kutzing ex Born. et Flah, Botrydium granulatum. (L.) Greville; Vaucheria geminata (Vaucher) DC. y Stichococcus bacilaris Naegeli, las mismas que se describen e ilustran.

\section{MATERIAL Y METODOS}

Las especies estudiadas forman parte de la colección de algas del Herbario San Marcos (USM) del Museo de Historia Natural "Javier Prado", las mismas que están preservadas en una solución de formol al $5 \mathrm{o}$.

Las observacones se hicieron en preparados simples y las ilustraciones se realizaron con el auxilio de la cámara clara. 
PARTE SISTEMATICA

CYANOPHYTA

HORMOGONALES

Oscillatoriaceae

Microcoleus Desmaziers, 1823

Microcoleus paludosus (Kutzing) Gomont.

(Figs. 1, 2 y 3

Filamentos simples o formando un estrato azul verde intenso, no ramificado, algunas veces divididos en la porción terminal; estuche mucilaginosos amplio, homogéneo, hialino, de superficie lisa y uniforme, con numerosos tricomas, rectos, constrictos a nivel de la pared transversal; células cilindricas, de 5 a 7 u de diámetro por 4 a 13 u de longitud, con protoplasto homogéneo verde azul brillante; célula apical cónica, no capitada.

Esta especie fue registrada frecuentemente en los bordes húmedos de los canales de riego de los jardines del Museo de Historia Natural y Jardin Botánico respectivamente.

Material estudiado: C. Acleto A-101, A-125, Museo de llistoria Natural "Javier Prado" y A-141 Jardin Botánico de la Universidad Nacional Mayor de San Marcos.

\author{
Nostocaceae \\ Nodularia Mertens, 1822 \\ Nodularia spumigena Mertens ex Born. et Flah.
}

Fig. 4

Filamentos libres o formando una masa mucosa, verde brillante; estuche mucilaginosos moderadamente engrosado, hialino y homogéneo; tricomas con células discoidales de 8 a 12 u de diámetro por 3 a 4 u de longitud; heterocistos del mismo tamaño que las células vegetativas, intercalares; aquinetos esféricos o comprimidos, de 12 a 15 u de diametro por 6 a $10 \mathrm{u}$ de longitud, solitariios o en series.

Forma una masa mucosa verde brillante en terrenos humedos.

Material estudiado: C. Acleto A-139, A-281 Museo de Historia Natural "Javier Prado".

Cylindrospermum Kutzing, 1843

Cylindrospermum licheniforme Kutzing ex Born. et Flah.

Figs. 5 y 6

Tricomas con células de longitud y diámetro iguales o cilíndricas, de 4 a 5 u de longitud, constrictas a nivel de la pared transversal, con protoplasto finamente granulado, azul verde pálido; heterocistos oblongos, de 7 a 12 u de longitud por 4 a 6 u de diámetro; aquinetos oblongos, de 10 a 38 u de longitud por 12 a 14 u de diámetro, con pared engrosada, lisa, marrón rojisa.

Habita en suelos húmedos, formando manchas de color verde oscuro brillante o plateadas.

Material estudiado: C. Acleto A-258, A-285 Museo de Historia Natural “Javier Prado".

CHRYSOPHYTA

XANTHOPHYCEAE

HETEROSIPHONALES

Botrydiaceae 
Botrydium Wallroth, 1815

Botrydium granulatum (L) Greville

Figs. 7,8 y 9

Planta cenocítica, con la porción aérea vesicular, subpiriforme ovoide, o globosa, alcanza más de $2 \mathrm{~mm}$ de diámetro y toda la planta más de $5 \mathrm{~mm}$ de longitud; verde brillante; la pared celular es delgada y granulada con impregnaciones de carbonato; la porción rizoidal carece de cromatóforos, con ramificaciones dicotómicas, progresivamente abundantes hacia la parte inferior. Hipnosporas numerosas, de diferentes tamaños, localizadas generaimente en la porción rizoidal, verde amarillentas a rojizas.

Habita en suelos húmedos de los jardines.

Material estudiado: C.Acleto A-129, A-212, A-213, A-215, A-216 y A-248 Museo de Historia Natural "Javier Prado"

\author{
Vaucheriaceae \\ Vaucheria DC., 1803 \\ Vaucheria geminata (Vaucher) DC
}

Fig. 10

Planta filamentosa, cenocítica, ramificada, entrecruzadas, de 81 a 108 u de diámetro, con numerosos cromatóforos discoidales, verde amarillento brillante; ramas fértiles constrictas en su base, con oogonio generalmente en pares, globosos o sublogosos, de 94 u de diámetro, con pared celular engrosada y abundarie sustancia de reserva; anteridio curvado o circinado, situado en el extremo distal de la ramá fértil, entre los oogonios.

Forma una capa afelpada verde brillante en suelos húmedos protegidos de los jardines o en los bordes de los canales de riego.

Material estudiado: C.Acleto A-217 Jardin Botánico, A-249 Museo de Historia Natural "Javier Prado", A-272 Urb. Apolo, La Victoria y A-282 Parque de la Reserva.

\title{
CHLOROPHYTA \\ ULTOTRICHALES \\ Ulotrichaceae \\ Stichococcus Naegeli, 1849
}

\section{Stichococcus bacilaris Naegeli}

Fig. 11

Filamentos cortos, de células cilíndricas, de 2 a 3.5 u de diámetro por 3 a 8 u de longitud, ligeramente constrictas a nivel de la pared transversal o frecuentemente disgregadas dando lugar a células bacilares libres, con cloroplasto laminar parietal, verde pálido. Habita en la superficie húmeda de los terrenos de cultivo.

Material estudiado: C. Acleto A-134 Jardin Botánico y A-163 terreno de cultivo próximo al Aeropuerto Internacional Lima - Callao.

\section{AGRADECIMIENTOS}

Agradezco al Dr. César Acleto, encargado de la Sección de Algas del Herbario San Marcos (USM). del Museo de Historia Natural "Javier Prado" por sugerirme el tema y por la ayuda brindada durante el desarrollo de este trabajo.

Al Dr. Ramón Ferreyra, Director del Museo de Historia Natural "Javier Prado" por haberme permitido el uso del laboratorio de la Sección Algas de dicha institución. 


\section{REFEENCIAS BIBLIOGRAFICAS}

DESIKACHARY , T.V. 1959. Cyanophyta. Indian Coun. Of Agric. Res. 686 pp. 139 pls., New Delhi.

DROUET, F. 1968. Revision of the clasification of the Oscillatoriaceae. The Acad. of Nat. Sci, of Philadelphia, Monograph 2 x $15: 1$ - 370, 131 figs.

PRESCOTT, G.W. 1962. Algae of th Western Great Lakes, Rev. ed. WM.C. Brown Co. Pub., Dubuque, Iowa.

SMITH, G.M. 1950. Fresh-water algae of the United States. wnd. ed. McGraw -Hill Book Co. New Yor.

TELL, G. 1970. Contribucion al conocimiento de las algas del suelo bonaerense. I. Botrydium granulatum y B. tuberosum Darwiniana 6: 140-143

TILDEN, Josephine 1910. Minnesota Alage. I. The Myxophyceae of the North America and adjacent region including Central America, Greenland, Bermuda, the West Indies and Hawai, iv +328 pp., 20 pls.

- VENKATARAMAN, G.S. 1961. Vaucheriaceae Indian Coun. of Agric. Rec. 112 pp., 74 figs. New Delhi.

\section{LEYENDA}

Figs. 1,2 y 3 Microcoleus paludosus (Kutzing) Gomont. Porción terminal de dos filamentos y detalle de una tricoma respectivamente.

Fig. 4. Nodularia spumigena Mertens ex Born. et Flah. Porción media de un filamento con heterocistos y aquinetos.

Figs. 5 y 6. Cylindrospermun licheniforme Kutzing ex Born. et Flah. Parte terminal de varios tricomas con heterocistos y aquinetos.

Figs.7, 8 y 9. Botrydium granulatum (L) Greville. Hipnosporas y células vegetativas respectivamente.

Fig. 10 Vaucheria geminata (Vaucher) DC. Características de las estructuras reproductivas.

Fig. 11 Stichococcus bacilaris Naegeli Individuos unicelulares y filamentos cortos de 2 células. 

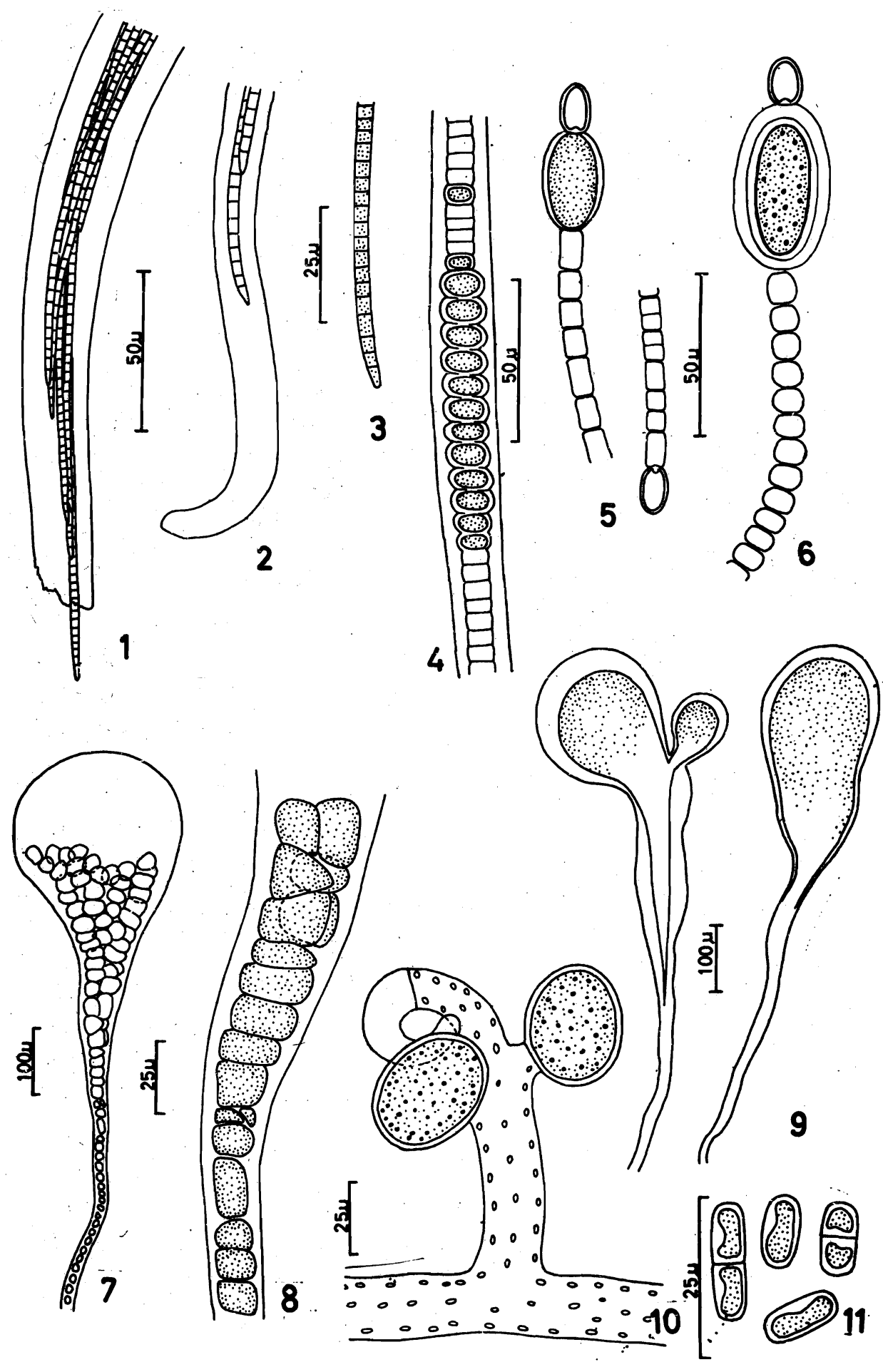